\title{
Coherent control of tunneling in a quantum dot molecule
}

\author{
J. M. Villas-Bôas, ${ }^{1,2}$ A. O. Govorov ${ }^{2}$ and Sergio E. Ulloa ${ }^{2}$ \\ ${ }^{1}$ Departamento de Física, Universidade Federal de São Carlos, 13565-905, São Carlos, São Paulo, Brazil \\ ${ }^{2}$ Department of Physics and Astronomy, Condensed Matter and Surface Science Program, \\ Ohio University, Athens, Ohio 45701-2979
}

\begin{abstract}
We demonstrate theoretically that it is possible to use Rabi oscillations to coherently control the electron tunneling in an asymmetric double quantum dot system, a quantum dot molecule. By applying an optical pump pulse we can excite an electron in one of the dots, which can in turn tunnel to the second dot, as controlled by an external voltage. Varying the intensity of the pulse one can suppress or enhance the tunneling between the dots for given level resonance conditions. This approach allows substantial flexibility in the control of the quantum mechanical state of the system.
\end{abstract}

Quantum dot (QD) structures provide a threedimensional confinement of carriers. Electrons and holes in the QD can occupy only a set of states with discrete energies, just as in an atom, and can thus be used to perform "atomic physics" experiments in solid state structures. One advantage of QDs is that they provide different energy scales and physical features which can be easily varied over a wide range of values. Most important, perhaps, is that QDs also allow the control of direct quantum mechanical electronic coupling with not only composition but externally applied voltages. These flexible systems represent therefore the ideal for theoretical and experimental investigations, where the interactions between light and matter can be studied in a fully controlled, well-characterized environment, and with excellent optical and electrical probes. These features make semiconductor QDs promising candidates for applications in electro-optical devices such as QD lasers, $\stackrel{1.2}{\stackrel{1}{1}}$ and in quantum information processing. ${ }^{3.4 .5,6}$ In the latter case, one can exploit the optical excitation in a $\mathrm{QD} \stackrel{3.5}{\$}$ or its spin state, .6 as qubits. These high expectations are produced by experimental advances in the coherent manipulation of QD states, such as the exciton Rabi oscillations in single dots, achieved by the application of electromagnetic pulses $\stackrel{78.9 .10 .11}{ }$ Coherent phenomena in ensembles of QDs have also been observed 12.13.14.15.16 Similarly, lithographically-defined dots have shown great potential in the control of coherently coupled systems $\frac{17.18 .19}{}$

The ability to assemble collections of QDs with designed geometries opens up a number of interesting possibilities. Here we describe theoretically the behavior of a QD-molecule formed from an asymmetric double QD system coupled by tunneling. Such a QD molecule can be fabricated using self-assembled dot growth technology ${ }^{20}$ By applying a near resonant optical pulse we can excite one electron from the valence to the conduction band in one dot, which can in turn tunnel to the second dot. We show that by suitably varying the frequency detuning or applied voltage on the QD pair, it is possible to control the inter-dot oscillations in the system, or use them to monitor the coherent state of one dot in the presence of the radiation field. This opens up the possibility of controlling the quantum mechanical state of such structure, perhaps useful in the field of quantum computation and information storage.

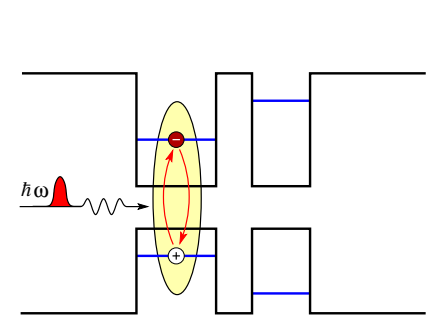

(a)
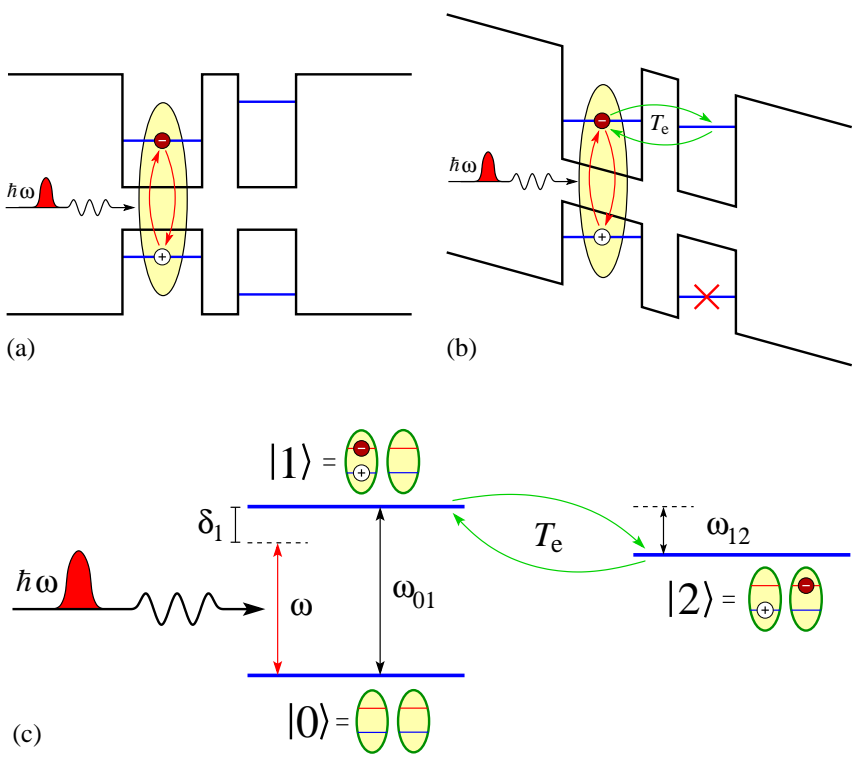

FIG. 1: (Color online) Schematic band structure and level configuration of a double QD system. (a) Without a gate voltage, electron tunneling is weak. (b) With applied gate voltage, conduction band levels get into resonance, increasing their coupling, while valence band levels become even more off-resonance, resulting in effective decoupling of those levels. (c) Levels taken into account by Hamiltonian model and the basis of states used to describe it. A pulsed laser excites one electron from the valence band that can tunnel to the other dot. We assume that the hole cannot tunnel in the time scale we are considering here.

A schematic representation of the Hamiltonian for our model can be seen in Fig. 1] In Fig. M(a), without a gate voltage, the levels are out of resonance, which results in weak interdot tunneling. In contrast, Fig. I(b) shows the situation with a gate voltage, where the conduction band levels get closer to resonance, greatly increasing their coupling, while the valence band levels get more off-resonance, resulting in effective decoupling of those levels. Considering this last configuration, we can neglect the hole tunneling and write the Hamiltonian as represented in Fig. 1(c). The electromagnetic field is introduced by the usual dipole interaction, which couples the ground state $|0\rangle$ (the system without excitations) with the exciton state $|1\rangle$ (a pair of electron and hole bound in the first dot) ${ }^{23}$ The electron tunneling, on the other 
hand, couples the exciton $|1\rangle$ with the indirect exciton state $|2\rangle$ (one hole in the first dot with an electron in the second dot). Using this configuration the Hamiltonian can be written $\operatorname{as}^{24}$

$$
\begin{aligned}
H= & \sum_{j} \varepsilon_{j}|j\rangle\langle j|+T_{e}(|1\rangle\langle 2|+| 2\rangle\langle 1|) \\
& +\hbar \Omega\left(e^{-i \omega t}|0\rangle\left\langle 1\left|+e^{i \omega t}\right| 1\right\rangle\langle 0|\right),
\end{aligned}
$$

where $\varepsilon_{j}$ is the energy of the state $|j\rangle, T_{e}$ is the electron tunneling matrix element, and $\Omega(t)=\langle 0|\vec{\mu} \cdot \vec{E}(t)| 1\rangle / 2 \hbar$, where $\vec{\mu}$, the electric dipole moment, describes the coupling to the radiation field of the excitonic transition, and $\vec{E}(t)$ is the pulse amplitude which can have different shapes ${ }^{25}$

To simplify the time-dependent Schrödinger equation, we utilize the unitary transformation

$$
|\psi\rangle=\exp \left[-\frac{i \omega t}{2}(|1\rangle\langle 1|-| 0\rangle\langle 0|+| 2\rangle\langle 2|)\right]\left|\psi^{\prime}\right\rangle
$$

which removes the fast oscillating term of the Hamiltonian. The resulting effective Hamiltonian can be written as (with $\hbar=1$ )

$$
H^{\prime}=\frac{1}{2}\left(\begin{array}{ccc}
-\delta_{1} & 2 \Omega & 0 \\
2 \Omega & \delta_{1} & 2 T_{e} \\
0 & 2 T_{e} & \delta_{2}
\end{array}\right),
$$

where $\delta_{1}=\omega_{01}-\omega$ is the detuning of the laser with the exciton resonance, $\delta_{2}=2 \omega_{12}+\delta_{1}$, and $\omega_{i j}=\left(\varepsilon_{i}-\varepsilon_{j}\right)$. Provided that all remaining parameters change slowly, or assuming that the pulse has a broad square shape, the time dependence of the effective Hamiltonian can be neglected, and the state vector of the system expressed as the superposition of the three eigenstates (or dressed states 21 ) of Hamiltonian (31). This problem has exact solution given by the roots of a cubic equation. An interesting case is when the pulse is in resonance with the exciton energy $\delta_{1}=0$ and the levels $|1\rangle$ and $|2\rangle$ are also in resonance, resulting $\delta_{2}=2 \omega_{12}=0$ (this value of $\omega_{12}$ can be tuned with an applied gate voltage). In this case the eigenvalues of Hamiltonian (3) have the simple form, $\lambda_{0}=0, \lambda_{ \pm}= \pm \sqrt{\Omega^{2}+T_{e}^{2}}$ with corresponding eigenstates

$$
\begin{aligned}
& \left|\lambda_{0}\right\rangle=\cos \theta|0\rangle-\sin \theta|2\rangle, \\
& \left|\lambda_{ \pm}\right\rangle=\frac{1}{\sqrt{2}}(\sin \theta|0\rangle \pm|1\rangle+\cos \theta|2\rangle),
\end{aligned}
$$

where $\cos \theta=T_{e} / \sqrt{\Omega^{2}+T_{e}^{2}}$.

Assuming that we start the system in the ground state $|0\rangle$, the occupation probability of the states of the Hamiltonian can be expressed as

$$
\begin{aligned}
& P_{0}(t)=\left|\sin ^{2} \theta \cos (\Theta t)+\cos ^{2} \theta\right|^{2}, \\
& P_{1}(t)=\sin ^{2} \theta \sin ^{2}(\Theta t), \\
& P_{2}(t)=\sin ^{2} \theta \cos ^{2} \theta|\cos (\Theta t)-1|^{2},
\end{aligned}
$$

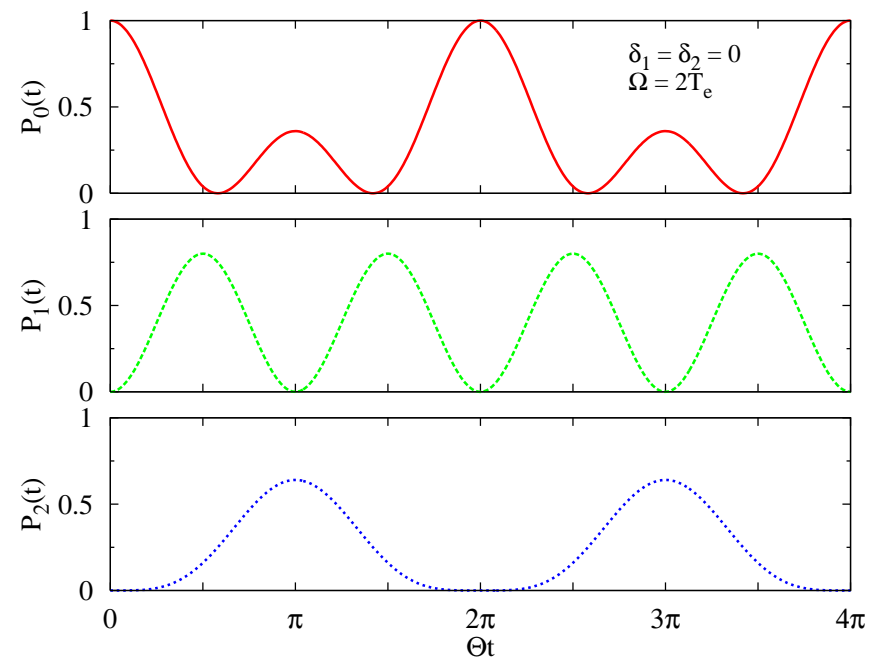

FIG. 2: (Color online) Population of the levels of the system as given by Eqs. (5). Note that at $\Theta t=n \pi$, for $n$ odd, the system does not fully restore the population of $|0\rangle$, as the system partially occupies state $|2\rangle$ if $T_{e} \neq 0$.

where $\Theta=\sqrt{\Omega^{2}+T_{e}^{2}}$. The result for $\Omega=2 T_{e}$ can be seen in Fig. 22 where we notice that the Rabi oscillations are incomplete, as interdot tunneling transfers some of the population to the indirect exciton state $|2\rangle$. Experimentally this could be very useful since one could monitor the population of the second dot as a non-disturbing probe of the coherent state of the QD. The presence of Rabi oscillations generated by the optical pulse in the first dot could then be directly measured. This can be done by sending a probe pulse at the resonance frequency of the exciton in the second (smaller) dot that has different (larger) frequency from the first and would then measure the transient differential transmission, reflecting its population. If the electron is in the small $\mathrm{QD}$, the resonant photon cannot be absorbed there, both because of Pauli blocking of the electron, and because Coulomb blockade interaction would require higher energy to create a charged exciton ${ }^{22}$ Another interesting possibility is to measure the photocurrent induced by the pulse in a double QD diode structure, similar to that used by

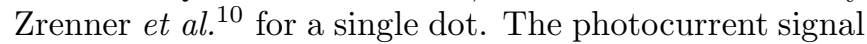
would be a direct measure of how much the electron has tunnelled to the second dot.

Although conceptually simple, the dynamics of the system presents some surprises that are not intuitive. For example, we would expect that the best way to create the state with one electron in the second dot, (state $|2\rangle$ in our model) would be to apply a pulse in resonance with the exciton energy, so that we can create one electronhole pair in the first dot and then allow the electron to tunnel to the second dot easily if its states are also in resonance $\left(\epsilon_{1}=\epsilon_{2}, \omega_{12}=0\right)$. This expectation is in part wrong, as we can see in Fig. 3 We plot there the average occupation of level $|2\rangle\left[1 / t_{\infty} \int_{0}^{t_{\infty}} P_{2}(t) d t\right]$ as a function of the voltage-controlled detuning of the levels $\epsilon_{1}$ and $\epsilon_{2}\left(\omega_{12}\right)$, for a resonant pulse in (a) and out of resonance in (b), assuming $\Omega=0.05 \omega$ and $T_{e}=0.01 \omega$. Notice that we have two equal peaks in (a), but neither 

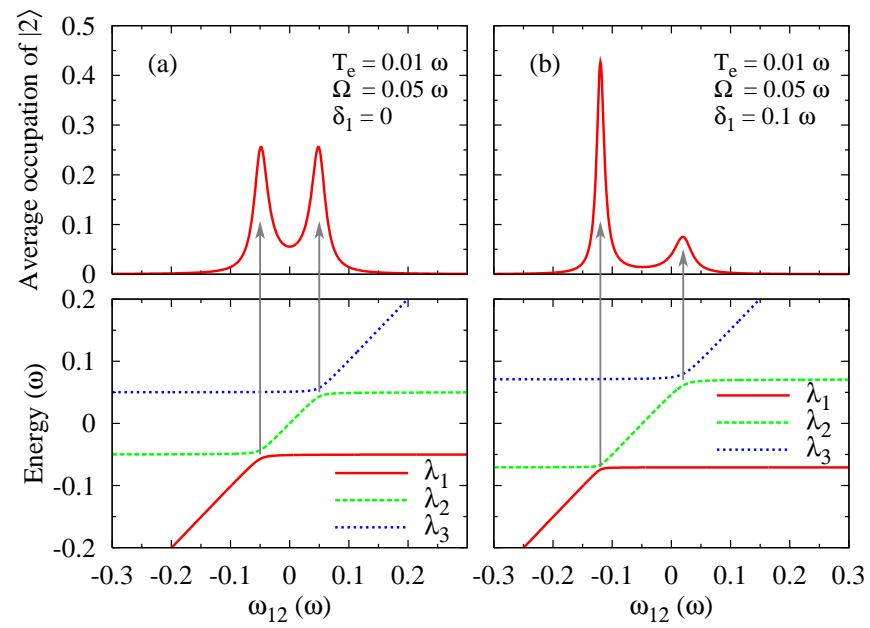

FIG. 3: (Color online) Average occupation of level $|2\rangle$ as function of detuning between levels $|1\rangle$ and $|2\rangle\left(\omega_{12}\right)$ for $T_{e}=0.01 \omega$ and $\Omega=0.05 \omega$. In (a) the pulse is applied in resonance with the exciton energy $\left(\delta_{1}=0\right)$ and $(\mathrm{b})$ with a detuning of $\delta_{1}=0.1 \omega$. In lower panels we show the respective dressed energy spectrum, $\lambda_{i}$ eigenvalues of the effective Hamiltonian (3). Arrows indicate that anticrossings in the spectrum yield an efficient population transfer to level $|2\rangle$.

one is located when the levels are in resonance $\left(\omega_{12}=0\right)$. A better transfer can be reached when both, the laser and the level detuning are different from zero, as we can see in Fig. 3(b), where we have a very narrow and high peak at $\omega_{12} / \omega \approx-0.12$. To understand this behavior, we plot the energy spectrum as a function of $\omega_{12}$ in the lower panels. We can see that the peaks in the average occupation of state $|2\rangle$ occur exactly at the anticrossing positions in the dressed spectrum, as indicated by arrows. An anticrossing indicates sizable mixing between levels from the same subspace of a symmetry group in Hilbert space. This mixing allow a maximum exchange of probability between the states involved. In our case this will reflect a maximum transfer of population to the level $|2\rangle$. Varying the gate voltage in an asymmetric double dot diode to tune the levels in and out of resonance would be an experimental implementation of this idea, resulting in peaks in the photocurrent at gate voltages satisfying the conditions above ${ }^{26}$

Another experimental possibility is to keep the gate voltage fixed (keep $\omega_{12}$ fixed) and vary the pulse frequency (vary the detuning of the laser with the exciton energy $\delta_{1}$ ) while measuring the induced photocurrent. The expected results can be seen in Fig. 4 Notice that when the levels of the different dots are in resonance $\left(\omega_{12}=0\right)$ the effective tunneling is weak for all range of detuning, re-enforcing the idea discussed above about the relatively poor electron transfer when the levels are in resonance. In contrast, the result for $\omega_{12} \neq 0$, present a clear sharp peak. This highly efficient transfer is likely to produce a clearly observable result in experiments. Incidentally, average occupation for $T_{e} \simeq \Omega$ results in strong transfer for $\delta_{1} \simeq-\omega_{12}$ (inset, Fig. (4).

We should emphasize that these results represent nothing but the control of the electron tunneling between dots using the coherent Rabi oscillation induced by the laser

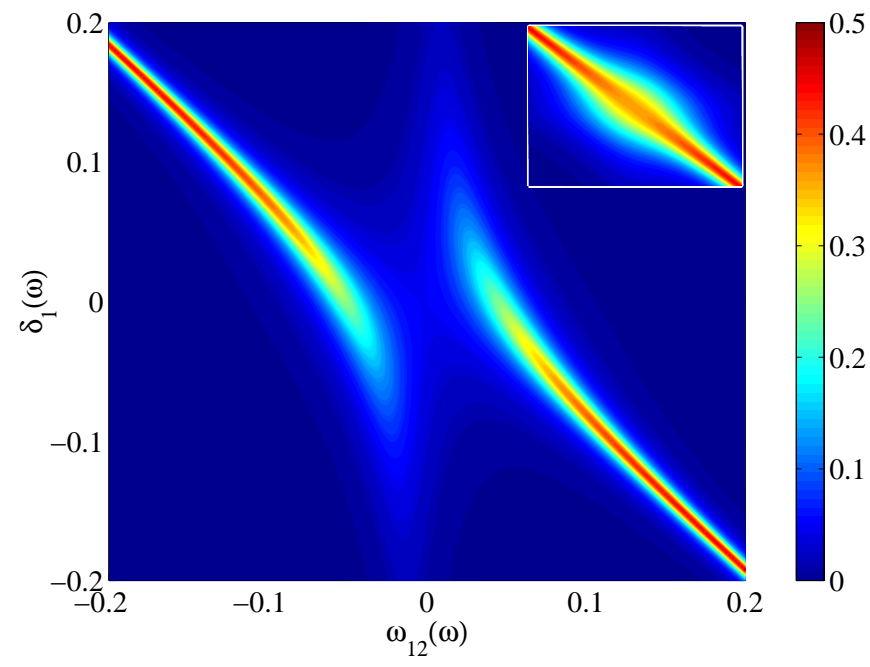

FIG. 4: (Color online) Average occupation of level $|2\rangle$ as function of laser detuning $\left(\delta_{1}\right)$ and level separation $\left(\omega_{12}\right)$ for $T_{e}=0.01 \omega$ and $\Omega=0.05 \omega$. Notice that if $\omega_{12}=0$ we have poor population transfer to state $|2\rangle$. The best result is obtained when both, the laser detuning $\left(\delta_{1}\right)$ and the levels separation $\left(\omega_{12}\right)$ are non-zero. Inset shows the result for $T_{e}=\Omega=0.05 \omega$.

in one of the dots and, in this way, the manipulation of the states of the system. One could imagine that such Rabi oscillation in an atomic molecule is also possible, but the experimental ability to control the interdot tunneling is unique to the QD system. This control could have a profound impact in the emergent field of quantum information processing.

Another parameter also experimentally tunable is the amplitude (or intensity) of the laser pulse, $\Omega$. It is interesting to note that for a given set of detuning and voltage values $\left(\delta\right.$ and $\left.\omega_{12}\right)$, the average occupation of state $|2\rangle$ is not a monotonic function of $\Omega$ : Figure 5 shows results for the average occupation of level $|2\rangle$ for a resonant pulse $\left(\delta_{1}=0\right)$ as a function of the pulse amplitude $\Omega$ when levels $|1\rangle$ and $|2\rangle$ are in and out of resonance. Note that when $\omega_{12}=0$, there is a peak exactly at the point where $\Omega=T_{e}$, as one would expect from a simple level mixing scheme, due to tunneling $T_{e}$ splitting the levels, and which are then effectively reconnected by the pulse $\Omega$. If we further increase the amplitude, we observe a suppression of the tunneling, where the average occupation drops basically to zero. On the other hand, if the levels are initially out of resonance, the tunneling is weak and it can be substantially enhanced by increasing the intensity of the pulse. Note for example, as indicated by the arrow in Fig. [5 that the tunneling for the out of resonance case is higher than the case for resonant levels. It is clear that larger level detuning $\omega_{12}$ requires a larger $\Omega$ to achieve optimal transfer, but it is nevertheless always achievable, even if the maximal amplitude is not as large as in the case $\omega_{12}=0$. (This situation changes in fact, and if $\delta_{1} \neq 0$, the maximum transfer to $|2\rangle$ occurs at finite $\omega_{12}$ values - not shown.)

We should be mindful that our solution here assumes constant $\Omega$, and is therefore valid in the case of slowly/smoothly-shaped pulses. Shape forming and pulse 


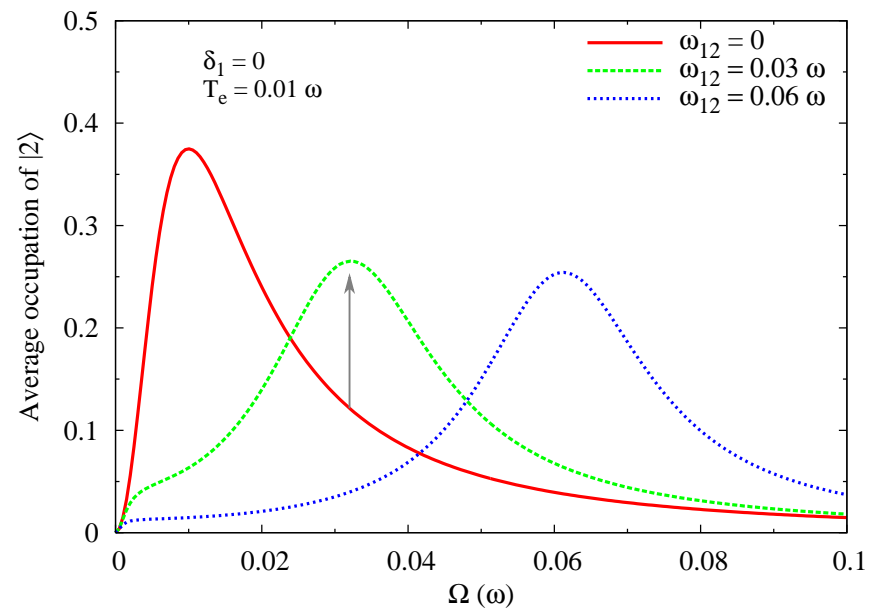

FIG. 5: (Color online) Average occupation of level $|2\rangle$ as a function of pulse amplitude $\Omega$ for the laser frequency in resonance with exciton energy $\left(\delta_{1}=0\right)$, and $T_{e}=0.01 \omega$. Solid line is the result for $\omega_{12}=0$, dashed line for $\omega_{12}=0.03 \omega$ and dotted line for $\omega_{12}=0.06 \omega$. Arrow indicates that for $\Omega \approx 0.03 \omega$, for example, there is an enhancement of the tunneling probability compared with the on-resonant case $\left(\omega_{12}=0\right)$. Notice also tunneling is suppressed if pulse has high amplitude.

sequence design give additional flexibility to control the quantum mechanical state of this QD molecule. We will report elsewhere our exploration of these degrees of freedom and the anticipated advantages to control the system, useful perhaps in the nascent field of quantum computing and QD optics 10.22

We have studied a system of two coupled QDs, where the tunnel coupling can be efficiently controlled and used to optically monitor the Rabi oscillations in the system. The model can be solved exactly for long constantamplitude pulses. The results show that we are able to control the tunneling by tuning the parameters of the system such as the pulse intensity, laser frequency, and gate voltage. Tunneling can be either enhanced or suppressed, depending on the conditions. This provides an electro-optical method to control the electron population of the second dot. Experimentally one could monitor the population of the second dot using a suitably tuned probe laser bean, which will reflect the Rabi oscillations generated by the original optical pump pulse. This opens the possibility to explore different coherent states of coupled dot systems and allow their use in novel quantum optics arrangements.

This work was partially supported by FAPESP and US DOE grant no. DE-FG02-91ER45334. We thank C. J. Villas-Bôas and N. Studart for helpful discussions.
1 O. B. Shchekin, G. Park, D. L. Huffaker, and D. G. Deppe, Appl. Phys. Lett. 77, 466 (2000).

${ }^{2}$ H. Saito, K. Nishi, and S. Sugou, Appl. Phys. Lett. 78, 267 (2001)

3 A. Ekert and R. Jozsa, Rev. Mod. Phys. 68, 733 (1996).

4 D. Loss and D. P. DiVincenzo, Phys. Rev. A 57, 120 (1998).

5 M. S. Sherwin, A. Imamoğlu, and T. Montroy, Phys. Rev. A 60, 3508 (1999).

6 A. Imamoğlu, D. D. Awschalom, G. Burkard, D. P. DiVincenzo, D. Loss, M. Sherwin, and A. Small, Phys. Rev. Lett. 83, 4204 (1999).

7 T. H. Stievater, X. Li, D. G. Steel, D. Gammon, D. S. Katzer, D. Park, C. Piermarocchi, and L. J. Sham, Phys. Rev. Lett. 87, 133603 (2001).

${ }^{8}$ H. Kamada, H. Gotoh, J. Temmyo, T. Takagahara, and H. Ando, Phys. Rev. Lett. 87, 246401 (2001).

${ }^{9}$ H. Htoon, T. Takagahara, D. Kulik, O. Baklenov, A. L. Holmes, Jr., and C. K. Shih, Phys. Rev. Lett. 88, 087401 (2002).

10 A. Zrenner, E. Beham, S. Stufler, F. Findeis, M. Bichler, and G. Abstreiter, Nature (London) 418, 612 (2002).

11 X. Li, Y. Wu, D. G. Steel, D. Gammon, T. H. Stievater, D. S. Katzer, D. Park, C. Piermarocchi, and L. J. Sham, Science 301, 809 (2003).

12 J. Kim, O. Benson, H. Kan, and Y. Yamamoto, Nature (London) 397, 500 (1999).

13 P. Michler, A. Kiraz, C. Becher, W. V. Schoenfeld, P. M. Petroff, L. Zhang, E. Hu, and A. Imamoğlu, Science 290, 2282 (2000).

14 B. E. Cole, J. B. Williams, B. T. King, M. S. Sherwin, and C. R. Stanley, Nature 410, 60 (2001).

15 P. Borri, W. Langbein, S. Schneider, U. Woggon, R. L. Sellin, D. Ouyang, and D. Bimberg, Phys. Rev. B 66,
081306 (2002).

16 M. Pelton, C. Santori, J. Vučković, B. Zhang, G. S. Solomon, J. Plant, and Y. Yamamoto, Phys. Rev. Lett. 89, 233602 (2002).

17 R. H. Blick, D. W. van der Weide, R. J. Haug, and K. Eberl, Phys. Rev. Lett. 81, 689 (1998).

18 T. H. Oosterkamp, T. Fujisawa, W. G. van der Wiel, K. Ishibashi, R. V. Hijman, S. Tarucha, and L. P. Kouwenhoven, Nature (London) 395, 873 (1998).

19 A. W. Holleitner, R. H. Blick, A. K. Hüttel, K. Eberl, and J. P. Kotthaus, Science 297, 70 (2002).

20 P. M. Petroff, A. Lorke, and A. Imamoğlu, Phys. Today, 54(5), 46 (2001).

21 C. Cohen-Tannoudji, J. Dupont-Roc, G. Grynberg, Atomphoton interactions (Wiley, New York, 1992).

22 D. Gammon and D. G. Steel, Phys. Today, 55(10), 36 (2002).

23 The biexciton state is far off resonance due the electronelectron interaction and can safely be neglected in our analysis for long pulses (requiring low intensity to achieve a $\pi$-pulse), as has been experimentally demonstrated for a single dot 7.8.9.10.11

24 Assuming a left-polarized pulse or linearly polarized light in rotation wave approximation (RWA), which can be applied here since the pulse intensity is much smaller than the separation of levels coupled and frequency of the pulse.

${ }^{25}$ Notice that direct coupling from $|0\rangle$ to $|2\rangle$ is neglected, as the dipole moment for that spatially indirect exciton will be vanishingly small.

${ }^{26}$ We should mention that the confined Stark effect will shift the energy levels in each dot in experiments, so that there is a slight gate voltage dependence on $\omega_{01}(V)$, resulting in a corresponding $\delta(V)$, easily compensated during system characterization. 